\title{
Factors affecting time until myocardial infarction in hypertension patients using truncated Cox regression
}

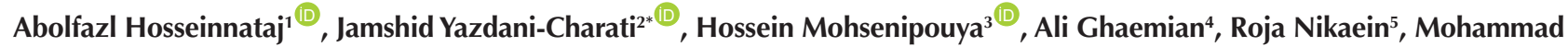 \\ Bagheri $^{6}$ \\ ${ }^{1} \mathrm{PhD}$ Candidate, Student Research Center, Mazandaran University of Medical Sciences, Sari, Iran \\ ${ }^{2}$ Department of Biostatistics, Cardiovascular Research Center, School of Health, Mazandaran University of Medical Sciences, \\ Sari, Iran \\ ${ }^{3}$ Health Education and Promotion, Faculty of Nursing, Mazandaran University of Medical Sciences, Sari, Iran \\ ${ }^{4}$ Department of Cardiovascular Disease, School of Medicine, Cardiovascular Research Center, Mazandaran University of \\ Medical Sciences, Sari, Iran \\ ${ }^{5}$ School of Health, Mazandaran University of Medical Sciences, Sari, Iran \\ ${ }^{6}$ Mazandaran University of Medical Sciences, Sari, Iran
}

*Corresponding Author: Jamshid Yazdani-Charati, Faculty of Health, Health Sciences Research Center, Mazandaran University of Medical Sciences, Sari, Iran, Tel: 0033242001, Email: Jamshid.charati@gmail.com

\begin{abstract}
Background and aims: Hypertension is one of the most common and important risk factors of cardiovascular diseases. This phenomenon, if not cured, causes lots of problems in vital organs of the body like kidneys, brain, eyes, and heart. The aim of the current study was to identify factors which affect the time until myocardial infarction (MI) in hypertension patients using truncated Cox regression.

Methods: This study was a population retrospective cohort and its statistical population included patients with hypertension symptoms, who had MI for the first time in Sari. A decision was made based on continuing the study as a nested case and control study because the number of $\mathrm{MI}$ incidents was low $(7 \%)$ in the studied sample. In addition, the Cox's model and truncated Cox were used for determining the factors affecting the patients' survival.

Results: The median and mean survival times in this sample were equal to 57 and 66.44 months, respectively. Considering time truncation, family history $(\mathrm{HR}=1.70)$ and the age at diagnosis $(\mathrm{HR}=0.99)$ were statistically significant in the Cox's model $(\mathrm{P}<0.05)$.

Conclusion: Overall, using the Akaike criterion for comparing the survival time without the effect of truncation, the Cox's model demonstrated better fitness by controlling the truncation effect. These results show the importance of controlling the truncation in those diseases in which it is impossible to distinguish the time of the appearance from the symptoms.

Keywords: Hypertension; Truncated Cox regression; Myocardial infarction; Survival time
\end{abstract}

Received: 2 September 2018, Accepted: 17 March 2019, ePublished: 30 August 2019

\section{Introduction}

The hypertension is considered as one of the most prevalent and essential risk factors of cardiovascular diseases, and simultaneously the rapid socio-economic changes during the last decade have spread various factors engaged in a cardiovascular disease like hypertension (1). This disease, if not treated, leads to different problems in vital organs of the body such as kidneys, brain, eyes, and heart. As one of the factors of coronary artery disease, hypertension can easily cause individual inabilities and death, and finally, heavy financial damages on society. However, this disease is controllable and thus its damages could be decreased considerably (2). For example, during the past 25 years, approximately $49 \%$ and $58 \%$ of death tolls caused by heart diseases and brain vascular disease, respectively, decreased because of on-time diagnosis and treatment in Europe and the United States (3). The World Health Organization (WHO) also estimated that more than 1.13 billion people in the world are affected by hypertension and about 7.1 million people die due to this abnormality every year. In 2015 , the prevalence of raised blood pressure in females (aged 18 and over) and males was around 20\% and 24\%, respectively (4). One-fifth of people in the United States is affected by hypertension that costs $\$ 20$ billion for the country every year (5).

Similarly, cardiovascular diseases are regarded as one of the most common abnormalities caused by hypertension (6). Nowadays, the death toll caused by coronary artery disease is averaged 34\% (about $40 \%$ for wealthy societies and $28 \%$ for low and intermediate societies) (5). In addition, nearly $27 \%$ of the death toll in more than 65-year-old population is due to cardiovascular diseases

(C) 2019 The Author(s); Published by Shahrekord University of Medical Sciences. This is an open-access article distributed under the terms of the Creative Commons Attribution License (http://creativecommons.org/licenses/by/4.0), which permits unrestricted use, distribution, and reproduction in any medium, provided the original work is properly cited. 
in the United States (6). Although the main cause of death toll in industrial countries, especially the United States and Western European countries, is related to heart diseases, these countries have passed the peak of heart and vessel diseases, and the death toll caused by this disease has decreased among these societies (7).

Hypertension is one of the key causes of the death toll in Iran as well. In 2015, 25\% of men and 17\% of women died in the middle age mostly owing to non-communicable diseases, including about a third from cardiovascular diseases (8). Further, this abnormality is the cause of $50 \%$ of the death toll among the people within the age range of 55-79 years old (9).

Coronary artery diseases lead to death, considerable inability, and productivity decreases, and thus bear high costs for health organizations (5). The level of the death toll caused by this phenomenon is growing in a wide variety of developing countries, especially Iran, because of sociocultural poverty about the risk factors of heart diseases and the lack of enough attention to disease prevention. Furthermore, coronary artery diseases cause more costs for these countries by a decrease in the incident age and the dangers of the incident in lower ages (10).

It should be noted that, based on the specialists' estimation, more than $80 \%$ of the early deaths related to heart diseases are preventable. Therefore, it is extremely important to diagnose this disease on time and treat it rapidly by conducting various studies in this regard (11).

In recent years, the statistical methods of survival analysis in scientific applies and theory studies are spreading, especially in medical research (12). In the current studies, the response variable is assumed as the time analysis until the event occurrence. The Cox' proportional hazard model is considered as a semi-parametric method and the Kaplan-Meier is regarded as a non-parametric method for analyzing the survival data. Despite some limitations, Cox's semi-parametric model is used as the most common model for modeling the factors affecting the survival. One of the most important features of the Cox proportional hazard model is that it does not depend on the probability distribution of survival time (13).

Incomplete observation is a special characteristic of the survival data and right censoring is regarded as the most popular form of incompleteness. For example, for some individuals, we only know that their true survival times are larger than certain censoring times (13). Another common form of incomplete observation is due to left truncation. In other words, some individuals are not followed from time zero, but only from later entry times (15). In numerous cohort studies, the patients are entered the study very late because of chronic and non-deathful nature of the disease (14). These types of data that have several criteria for their entrance into the study are called "truncated data" (14). The truncation may happen in different subjects like astrology, medicine studies, social sciences, criminology, economics, and the like.

The individual's entrance into the study depends on some factors before the event occurrence. The individuals' real survival time is more than the observed survival time if they fail to follow up from the first symptoms of the disease until the first observation and thus the data are called "left truncated" (15). In this kind of data, the individuals cannot experience the event before the time of the first observation. Therefore, the assumption is considered as not to be endangered (14). Most survival studies consider the only right-censored when the data are delayed to enter the study. In this study, the inclusion criteria were patients with hypertension although the patients entered the study by a delay because the onset of hypertension is unknown. The results of numerous studies demonstrated the effects of hypertension on myocardial infarction (MI), therefore, the current study sought to determine the risk factors of MI in patients with hypertension to consider both rightcensored and left truncation in order to increase their life quality while decreasing their medical expenses.

\section{Methods}

This study was a population retrospective cohort and its statistical population encompassed a group of patients who referred to Hazrat Abolfazl Medical Clinic of Sari (located in Northern part of Iran) given the entrance criterion of "hypertension" and the interest event as the first time MI without any prior experience. This center is a general medical clinic and is used to screen for hypertension patients. Therefore, the checklist of the clinic was the data collection instrument. The studied variables include the age, weight, height, body mass index (BMI), marital status, the number of children, habitation, the level of education, employment, smoking, as well as a history of MI and diabetes in the family, the consumption of blood pressure drugs, physical activity, and the abdomen fat. The samples were selected from among the patients' recorded files by using a random number table. This study had an observer as well. In the studied sample, the number of MIs were observed less than natural norms and therefore, some problems existed regarding calculating the survival time median and the factors affecting the MI. Accordingly, it was decided to continue the study as case-control research and to utilize a nested sampling technique. To this end, all individuals experiencing MI (25 patients) were considered as the cases and the random sample was selected among the individuals without any MI and its size double the cases (50 individuals) were regarded as controls. The data were analyzed by SPSS (version 16) and SAS (version 9.1) software. Similarly, the patients' survival of time (the time of hypertension disease occurrence until the first MI or the time of the study completion) was calculated based on the months. In addition, the Cox's model was used to determine the factors which affect the patients' survival. Figure 1 shows the steps of this study. Furthermore, Cox's 


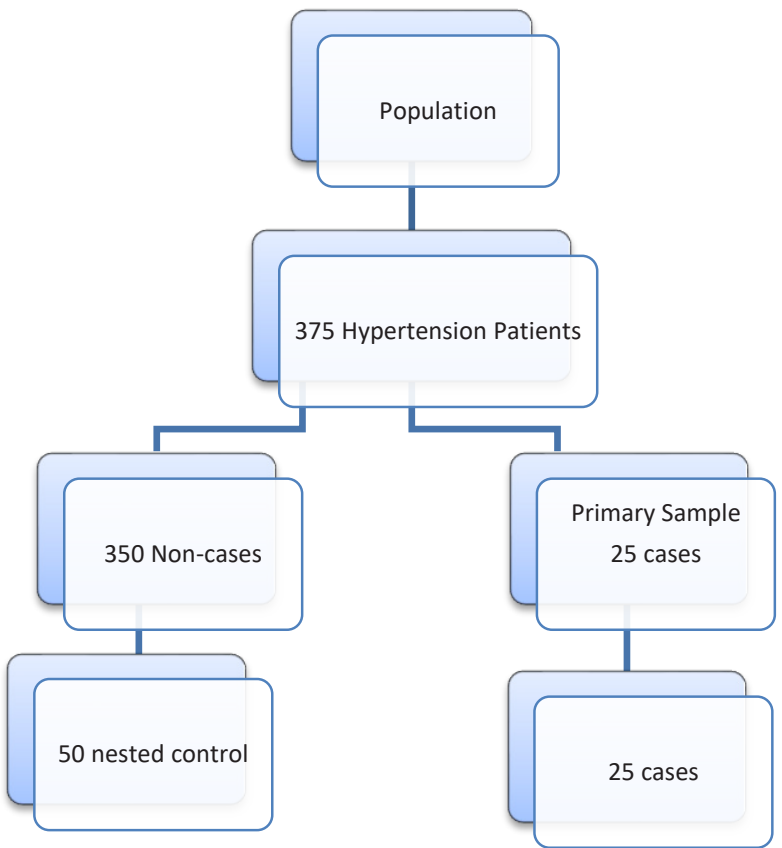

Figure 1. Steps of Study Design.

model both with and without truncation effects were compared according to Akaike criterion in order to test the data truncation effect. Moreover, every variable with a $P$ value of less than 0.3 in multiple analysis was entered into the multivariate model for analysis. In the current study, the patients who experienced no MI until the end of the study were considered as the right-censored and the significance level was equal to 0.05 .

Cox's semi-parametric regression was employed to assess the factors affecting the patients' survival of time (the time of hypertension disease occurrence until the first MI or the time of the end of the study) to determine the relationship with the probability of survival in all subgroups of the population. These subgroups included cholesterol status, age, weight, height, BMI, marital status, the number of children, habitation, the level of education, employment, and cigar smoking, as well as diabetes and MI history in the family, the consumption of blood pressure drugs, physical activity, and abdomen fat. The hazard ratio was calculated as well. $T$ was considered as the time of hypertension disease occurrence until the first MI or the time of the study end. Then, $T$ value could be thought to have a probability distribution. The main form of this model was as follows.

$\ln (h(T))=\ln \left(h_{0}(T)\right)+\sum_{i=1}^{p} x_{i} \beta_{i}$

where $\mathrm{x}_{1}, \ldots, \mathrm{x}_{\mathrm{p}}$ and $\beta_{1}, \ldots, \beta_{\mathrm{p}}$ are covariates and estimated regression coefficients, respectively, and $h_{0}(T)$ denotes the baseline hazard rate when all covariates are equal to zero (15). First, the Kaplan-Meier plot was used for treating the delay time and comparing the result by the log-rank test.
Similarly, log-rank (Mantel-Cox) test of the equality of survival distributions was utilized to analyze the significance of the survival difference between the categorical variables. $P<0.3$ was considered statistically significant for single variable analysis. Then, Cox proportional hazards regression analysis was performed to evaluate the impact of age, gender, nationality, the place of residence, the conditions of imprisonment, the date of diagnosis, the type of disease (e.g., pulmonary and extrapulmonary), HIV status, and treatment delay. Statistical analyses were conducted using SPSS software, version 20 and the value of $\alpha=0.05$ was considered statistically significant.

Results

The medical records of 375 patients affected by hypertension were investigated in the current study. Due to few numbers of events $(7 \%)$ in the sample under investigation and its related problems because of survival calculation and its affective factors, it was decided to conduct a case and control sampling for the studied data (case-cohort study). Thus, 25 patients (those with MI) were considered as cases and 50 other patients without any infarction occurrence as the controls, followed by analyzing these data. Table 1 represents the descriptive statistics and significant test between the variables and the event in the nested sample.

The mean duration of follow-up was 41.3 months. Based on the Kaplan-Meier estimate, the survival time mean and median in the nested sample were equal to 66.44 and 57 months for the hypertension patients, respectively. The results of the Kaplan-Meier estimate and hazard function plot are shown in Table 2 and Figure 2.

The log-rank test was applied to determine the important factors in the survival of hypertension patients. The results of this test showed that variables like habitation location, diabetes history, MI family history, drug consumption, blood lipids history, and marital status had a significant effect on the MI in patients with hypertension disease.

Cox's regression model is one of the methods for studying the effect of independent variables on survival time. By using the Cox proportional hazards model without controlling the truncation effect, the variables of MI history in first-degree relatives, as well as blood lipids and marriage statue were significant in the multiple analysis. The results are provided in Table 3.

Controlling the other variables, the myocardial history in first-degree relatives increased the hazard of MI by 4.68 times.

Moreover, the hazard of infarction was 16.35 times more in patients with high levels of blood lipids compared to those without blood lipids. Meanwhile, the hazard of infarction in married patients was 0.83 less than bachelor (single) patients.

In this model, the Akaike criterion was equal to 128.38 . Based on the table output, no significant relationship 
Table 1. Descriptive statistics and significant test

\begin{tabular}{|c|c|c|c|c|}
\hline Qualitative variables & Level & Case & Control & $P$ value (Chi-square) \\
\hline \multirow{2}{*}{ Sex } & Male & 10 & 25 & \multirow{2}{*}{0.47} \\
\hline & Female & 15 & 25 & \\
\hline \multirow{2}{*}{ Location } & Urban & 1 & 9 & \multirow{2}{*}{0.15} \\
\hline & Rural & 24 & 41 & \\
\hline \multirow{4}{*}{ Marital status } & Married & 18 & 43 & \multirow{4}{*}{0.04} \\
\hline & Single & 30 & 3 & \\
\hline & Divorced & 1 & 1 & \\
\hline & Other & 36 & 3 & \\
\hline \multirow{2}{*}{ Education } & Illiterate & 21 & 33 & \multirow{2}{*}{0.08} \\
\hline & Literate & 4 & 17 & \\
\hline \multirow{3}{*}{ Job } & Employee & 9 & 16 & \multirow{3}{*}{0.33} \\
\hline & Self-employment & 2 & 11 & \\
\hline & Unemployed & 14 & 23 & \\
\hline \multirow{3}{*}{ Physical activity } & Regular & 0 & 4 & \multirow{3}{*}{0.05} \\
\hline & Irregular & 3 & 12 & \\
\hline & No & 22 & 34 & \\
\hline \multirow{2}{*}{ Smoking } & Yes & 1 & 4 & \multirow{2}{*}{0.66} \\
\hline & No & 24 & 46 & \\
\hline \multirow{2}{*}{ Diabetes history } & Yes & 3 & 15 & \multirow{2}{*}{0.08} \\
\hline & No & 22 & 35 & \\
\hline \multirow{2}{*}{ History in family } & Yes & 6 & 3 & \multirow{2}{*}{0.03} \\
\hline & No & 19 & 47 & \\
\hline \multirow{2}{*}{ Medicine consumption } & Yes & 21 & 22 & \multirow{2}{*}{0.001} \\
\hline & No & 4 & 28 & \\
\hline Quantitative variables & & & & $P$-value (t-test) \\
\hline Age & & $63.48 \pm 10.99$ & $53 \pm 11.07$ & $<0.001$ \\
\hline BMI & & $28.22 \pm 5.02$ & $28.88 \pm 4.47$ & 0.56 \\
\hline Abdomen fat & & $108.56 \pm 8.98$ & $97.81 \pm 10.41$ & $<0.001$ \\
\hline Number of children & & 3.30 & 4.04 & - \\
\hline
\end{tabular}

Data are shown as number or mean $\pm \mathrm{SD}$.

BMI: Body mass index.

Table 2. The survival time mean and median for patients with hypertension by using the Kaplan-Meier method (time in months)

\begin{tabular}{|c|c|c|c|c|c|c|c|}
\hline \multirow{3}{*}{ Estimate } & \multicolumn{3}{|c|}{ Mean } & \multicolumn{4}{|c|}{ Median } \\
\hline & \multirow{2}{*}{ Standard Error } & \multicolumn{2}{|c|}{$95 \% \mathrm{Cl}$} & \multirow{2}{*}{ Estimate } & \multirow{2}{*}{ Standard Error } & \multicolumn{2}{|c|}{$95 \% \mathrm{Cl}$} \\
\hline & & Lower Bound & Upper Bound & & & Lower Bound & Upper Bound \\
\hline 66.46 & 6.25 & 54.18 & 78.70 & 57 & 11.81 & 33.85 & 80.15 \\
\hline
\end{tabular}

was observed between the age, sex, employment status, education statue, the number of children, diabetes history, medicine consumption, BMI, smoking, abdomen fat, and physical activity and the first MI occurrence in patients with hypertension.

The time of the first referral of the patients to the clinic was considered to be equal to the time of the entrance in the study because the exact time of hypertension disease occurrence was undistinguished in patients under investigation and therefore, the data related to the survival time were defective. Likewise, the left truncation was used as the patients' entrance into the study since the real survival time was bigger than the observed survival time.

Table 4 demonstrates variables which had a significant effect on the survival in the multiple Cox's model by controlling the truncation effect.

Based on the obtained data, patients with a family history of MI and younger patients were more likely to be afflicted with MI. The myocardial history in first-degree relatives increased the hazard of MI by $70 \%$. In addition, the risk of MI reduced by $1 \%$ for each year increase in the age and the Akaike criterion was calculated as 68.93 for this model.

\section{Discussion}

Today, the exact time of the onset of many diseases is still unknown and the disease is only diagnosed if a symptom is observable. Therefore, it is required to pay attention to the risk factors of the disease. In the survival analysis, which is the time onset of the disease until the desired outcome, it is necessary to control the time of entry into the study. Two strategies can be considered for the time of entry, including the time at birth as the point of origin and the truncation time. The subjects are not followed from birth 


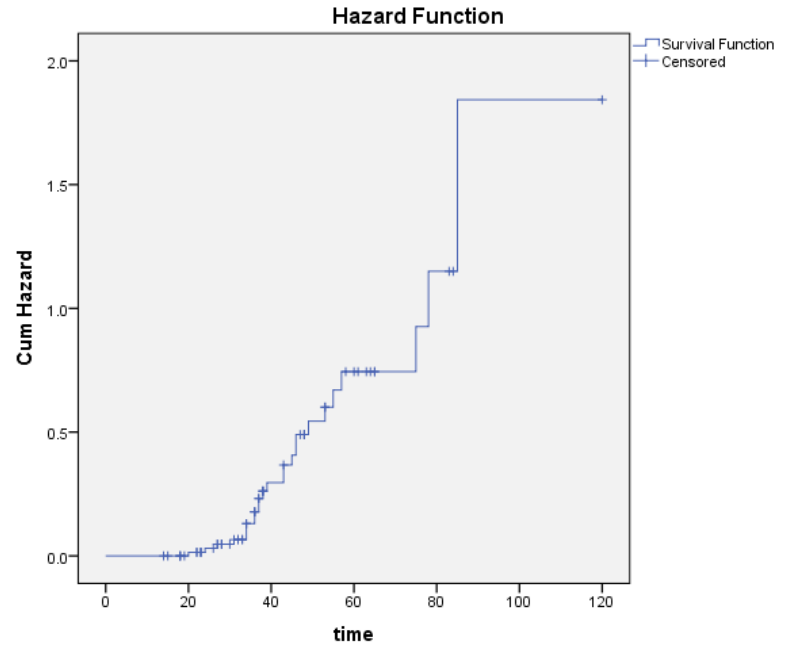

Figure 2. The Kaplan-Meier estimate of cumulative hazard function plot (time in month).

and those subjects who die before reaching the recruitment age have no chance to be sampled, and therefore age at birth is less commonly used for this purpose. In this paper, the left truncation was used to control the time of entry into the study.

In the current investigation, $25(6.7 \%)$ patients were afflicted with MI and the male/female ratio was estimated at 0.73 that was less than the results of the other similar investigation (16).

In this study, the age group of 41-60 was the most common age incidence. In addition, the age mean of patients was totally 56.80 , as well as 55.26 and 58.15 years for male and female patients with hypertension, respectively, which was less than the estimated result of the other similar studies (17).

The family history was determined as an effective factor on the survival in MI occurrence and there was more danger of MI in patients with a family history of MI in first-grade family members.

Based on the findings of several previous studies conducted in Britain (18), Brazil (19), and Iran (20), the family history was reported as a vital variable in heart attack occurrence. The results of this study regarding both models with and without controlling the truncation effect revealed that the hazard of MI was more in patients with hypertension due to their family history compared to the other patients. This could be attributed to several causes such as genetics, lifestyle, and a similar diet in the members of a family. The existence of heart disease family history is considered as a hazard factor for other members of family thus the on-time diagnosis and treatment of this endangered members are considered as the most essential factors of a decrease in the death toll of MI.

Recently, many experts and non-experts have cast doubt on the role of cholesterol as a risk factor for cardiovascular disease. Further, the results of various studies performed in countries like Brazil (19), Germany (10), and Iran (21) demonstrated that the blood lipid was regarded as an effective factor in MI. In this study, blood lipid had a significant effect on MI in the model without control left truncation. The results of this study indicated the important role of controlling the blood lipid factor in preventing heart diseases. Modern dietary recommendations indicate that a low-fat approach is useful for reducing the risk of MI (22).

The marital status was introduced as another predictive factor for MI occurrence in patients with hypertension (23). The hazard of infarction for unmarried individuals was calculated 5.9 times more than that of the married people. In a previous study conducted in this field, it was shown that the marital status was found as an effective factor in MI. This may be attributed to the effects of marriage on sanity healthy and life motivation and family supports about individual's health (24). However, the age factor represented no effect on patients' survival, which contradicts the results of other studies $(18,25)$. It was shown that the rate of survival increases by $0.01 \%$ in patients with later diagnosis (about one year). This is probably due to the low age mean of the studied patients compared to the other studies or, for example, not considering age as a qualitative variable since the infarction hazard increases until the age of 40 and decreases afterward.

Table 3. Significant variables in multiple Cox regression without truncation effect

\begin{tabular}{|c|c|c|c|c|c|}
\hline \multirow{2}{*}{ Variable } & \multirow{2}{*}{ Hazard Ratio } & \multirow{2}{*}{ Standard Error } & \multirow{2}{*}{$P$ Value } & \multicolumn{2}{|c|}{$95 \% \mathrm{Cl}$} \\
\hline & & & & Lower Bond & Upper Bound \\
\hline Family history & 4.68 & 2.81 & 0.01 & 1.44 & 15.22 \\
\hline Diagnosis age & 16.35 & 14.31 & 0.001 & 2.94 & 90.17 \\
\hline Marital status & 0.17 & 0.11 & 0.11 & 0.04 & 0.65 \\
\hline
\end{tabular}

Table 4. Significant variables in multiple Cox regression with truncation effect

\begin{tabular}{|c|c|c|c|c|c|}
\hline \multirow{2}{*}{ Variable } & \multirow{2}{*}{ Hazard Ratio } & \multirow{2}{*}{ Standard Error } & \multirow{2}{*}{$P$ Value } & \multicolumn{2}{|c|}{$95 \% \mathrm{Cl}$} \\
\hline & & & & Lower Bond & Upper Bound \\
\hline Family history & 1.70 & 0.23 & 0.02 & 1.24 & 2.15 \\
\hline Diagnosis age & 0.99 & 0.002 & 0.03 & 0.98 & 1.00 \\
\hline
\end{tabular}




\section{Conclusion}

In the multiple Cox's regression model without controlling the left truncation effect, a family history of MI, blood lipid, and marriage statue were found significant. However, the family history of infarction and age were significant in the multiple Cox's model with controlling the left truncation effect. After comparing these two models by using the Akaike criterion, Cox's model with controlling the truncation effect showed better fitness. Therefore, this result shows the importance of truncation control in patients with an undetermined time of symptom initiation.

It can cause bias if ignored delayed time enters the study.

\section{Conflict of interests}

None.

\section{Ethical considerations}

This study was approved by the Clinical Research Ethics Committee of the Mazandaran University of Medical Sciences under the code of IR.MAZUMS.REC.1394.1439.

\section{Acknowledgments}

The authors thank the Hazrat Abolfazl Clinic of Sari (Northern Iran), Mazandaran University of Medical Sciences, Iran, and all the patients who took part in this research. This study was funded by the Research Deputy of Mazandaran University of Medical Sciences (grant number 1439).

\section{References}

1. Kritz-Silverstein D, Laughlin GA, McEvoy LK, Barrett-Connor E. Sex and age differences in the association of blood pressure and hypertension with cognitive function in the elderly: The rancho bernardo study. J Prev Alzheimers Dis. 2017;4(3):16573. doi: 10.14283/jpad.2017.6.

2. Rashidi Y, Manaflouyan H, Pournaghi Azar F, Nikniaz Z, Nikniaz L, Ghaffari S. Knowledge, attitude and practice of Iranian hypertensive patients regarding hypertension. J Cardiovasc Thorac Res. 2018;10(1):14-9. doi: 10.15171/jcvtr.2018.02.

3. Sarikhani Y, Heydari ST, Emamghorashi F, Jafari F, Tabrizi $\mathrm{R}$, Karimpour $\mathrm{S}$, et al. Associated factors and standard percentiles of blood pressure among the adolescents of Jahrom City of Iran, 2014. Int J Pediatr. 2017;2017:3804353. doi: $10.1155 / 2017 / 3804353$.

4. WHO. Global strategy for women's, children's and adolescents' health (2016-2030). https://www.who.int/life-course/partners/ global-strategy/en.

5. Smeltzer SC, Bare BC, Hinkle JL, Cheever KH. Brunner \& Suddarth's textbook of medical-surgical nursing. Lippincott Williams \& Wilkins; 2010.

6. Malani PN. Harrison's principles of internal medicine. JAMA. 2012;308(17):1813-4. doi: 10.1001/jama.308.17.1813-b.

7. Stecker EC, Reinier K, Marijon E, Narayanan K, Teodorescu C, Uy-Evanado A, et al. Public health burden of sudden cardiac death in the United States. Circ Arrhythm Electrophysiol. 2014;7(2):212-7. doi: 10.1161/circep.113.001034.

8. Pagan E, Chatenoud L, Rodriguez T, Bosetti C, Levi F, Malvezzi $\mathrm{M}$, et al. Comparison of trends in mortality from coronary heart and cerebrovascular diseases in north and South America: 1980 to 2013. Am J Cardiol. 2017;119(6):862-71. doi: 10.1016/j. amjcard.2016.11.040.

9. Armas Rojas N, Dobell E, Lacey B, Varona-Pérez P, Burrett JA, Lorenzo-Vázquez E, et al. Burden of hypertension and associated risks for cardiovascular mortality in Cuba: a prospective cohort study. Lancet Public Health. 2019;4(2):e107-e15. doi: 10.1016/ s2468-2667(18)30210-x.

10. Tselios K, Gladman DD, Harvey P, Akhtari S, Su J, Urowitz MB. Abnormal Cardiac Biomarkers in Patients with Systemic Lupus Erythematosus and No Prior Heart Disease: A Consequence of Antimalarials? J Rheumatol. 2019;46(1):64-9. doi: 10.3899/ jrheum. 171436.

11. Dennis L Kasper DL, Harrison TR. Harrison's principles of internal medicine. New York: McGraw-Hill; 2005.

12. Ahmadi A, Soori H, Mehrabi Y, Etemad K, Khaledifar A. Epidemiological pattern of myocardial infarction and modelling risk factors relevant to in-hospital mortality: the first results from the Iranian Myocardial Infarction Registry. Kardiol Pol. 2015;73(6):451-7. doi: 10.5603/KP.a2014.0230.

13. Gosho M, Sato Y, Nagashima K, Takahashi S. Trends in study design and the statistical methods employed in a leading general medicine journal. J Clin Pharm Ther. 2018;43(1):3644. doi: 10.1111/jcpt.12605.

14. YuT, Wu L, Gilbert P. New approaches for censored longitudinal data in joint modelling of longitudinal and survival data, with application to HIV vaccine studies. Lifetime Data Anal. 2019;25(2):229-58. doi: 10.1007/s10985-018-9434-7.

15. Montaseri M, Yazdani Cherat J, Espahbodi F, Mousavi SJ. Five-year survival rate in hemodialysis patients Attending Sari Imam Khomeini Hospital. Journal of Mazandaran University of Medical Sciences. 2013;23(101):78-85. [Persian].

16. Cremer P, Nagel D, Mann H, Labrot B, Muller-Berninger R, Elster $\mathrm{H}$, et al. Ten-year follow-up results from the Goettingen Risk, Incidence and Prevalence Study (GRIPS). I. Risk factors for myocardial infarction in a cohort of 5790 men. Atherosclerosis. 1997;129(2):221-30. doi: 10.1016/s0021-9150(96)06030-3.

17. Mosa Farkhani E, Baneshi MR, Zolala F. Survival rate and its related factors in patients with acute myocardial infarction. Medical Journal of Mashhad University of Medical Sciences. 2014;57(4):636-46. [Persian].

18. Hippisley-Cox J, Coupland C, Brindle P. Development and validation of QRISK3 risk prediction algorithms to estimate future risk of cardiovascular disease: prospective cohort study. BMJ. 2017;357:j2099. doi: 10.1136/bmj.j2099.

19. Piegas LS, Avezum A, Pereira JC, Neto JM, Hoepfner C, Farran JA, et al. Risk factors for myocardial infarction in Brazil. Am Heart J. 2003;146(2):331-8. doi: 10.1016/s00028703(03)00181-9.

20. Sadeghi M, Ahmadi A, Baradaran A, Masoudipoor N, Frouzandeh S. Modeling of the relationship between the environmental air pollution, clinical risk factors, and hospital mortality due to myocardial infarction in Isfahan, Iran. J Res Med Sci. 2015;20(8):757-62. doi: 10.4103/1735-1995.168382.

21. Zahra PM, Azizollah AS, Masoud R, Hamed S, Mehrdad H, Ebrahim E. Coronary artery disease in critical patients of Iran. J Pak Med Assoc. 2012;62(12):1282-5.

22. Forouhi NG, Krauss RM, Taubes G, Willett W. Dietary fat and cardiometabolic health: evidence, controversies, and consensus for guidance. Bmj. 2018;361:k2139. doi: 10.1136/ bmj.k2139.

23. Wang J, Sun W, Wells GA, Li Z, Li T, Wu J, et al. Differences in prevalence of hypertension and associated risk factors in urban and rural residents of the northeastern region of the People's Republic of China: A cross-sectional study. PLoS One. 2018;13(4):e0195340. doi: 10.1371/journal.pone.0195340.

24. Shang YX, Ding WQ, Qiu HY, Zhu FP, Yan SZ, Wang XL. Association of depression with inflammation in hospitalized patients of myocardial infarction. PakJ Med Sci. 2014;30(4):6927.

25. Pejtersen JH, Burr H, Hannerz H, Fishta A, Hurwitz Eller N. Update on work-related psychosocial factors and the development of ischemic heart disease: a systematic review. Cardiol Rev. 2015;23(2):94-8. doi: 10.1097/ crd.0000000000000033. 